\title{
A29 THE EARLY MARGINAL ZONE B CELL INITIATED T INDEPENDENT TYPE 2 RESPONSE RESISTS THE PROTEASOME INHIBITOR BORTEZOMIB
}

V Lang, ${ }^{1}$ D Mielenz, ${ }^{2}$ H Martin Jäck, ${ }^{2}$ K Neubert, ${ }^{1}$ C Böhm, ${ }^{1,3}$ G Schett, ${ }^{3}$ R E Voll, ${ }^{1,3}$ S Meister ${ }^{1} 1$ Interdisciplinary Center for Clinical Research, Research Group $N^{2}$, Nikolaus Fiebiger-Center ofMolecular Medicine, University Hospital Erlangen, Erlangen, Germany; ${ }^{2}$ Division of Molecular Immunology, Department of Internal Medicine ${ }^{3}$, University of Erlangen-Nuremberg, Erlangen, Germany; ${ }^{3}$ Department of Internal Medicine ${ }^{3}$ and Institute of Clinical Immunology, University of Erlangen-Nuremberg, Erlangen, Germany

10.1136/ard.2010.129585c

The proteasome inhibitor bortezomib is clinically approved for the treatment of multiple myeloma. Recently, we demonstrated that bortezomib eliminates autoreactive plasma cells in SLE mice, thereby representing a promising novel treatment for antibody-mediated diseases. Here, we investigated the effect of bortezomib on the developing and pre-existing $T$ dependent antibody response towards dinitrophenylated keyhole limpet hemocyanin and the $T$ independent type 2 response towards NIP-Ficoll in BALB/c mice. Bortezomib treatment strongly reduced $\mathrm{T}$ dependent antibody titres mainly due to depletion of plasma cells. In contrast, the early $\mathrm{T}$ independent type 2 response, predominantly initiated by marginal zone (MZ) B cells, resisted bortezomib. Immunoproteasomal subunits and the antiapoptotic unfolded protein response were induced in NIP-Ficoll-stimulated MZ B cells after bortezomib treatment, but not in plasma cells. This induction might be cell autonomous and not a consequence of the microenvironment since mobilisation of $M Z B$ cells out of the spleen did not render them susceptible towards bortezomib. We conclude that the resistance of $M Z B$ cells against bortezomib leaves early $T$ independent responses protecting against bloodborne pathogens largely intact. This fact may account for a relatively low risk of bacterial infections compared to most other immunosuppressants and cytotoxic drugs. 